\title{
Indocyanine Green-Enhanced Thermotherapy for Retinoblastoma
}

\author{
Christiane E. Al-Haddad ${ }^{a} \quad$ Marwan Abdulaal $^{a} \quad$ Raya H. Saab ${ }^{b}$ \\ Ziad F. Bashshura \\ Departments of a Ophthalmology and ${ }^{b}$ Pediatric and Adolescent Medicine, American \\ University of Beirut Medical Center, Beirut, Lebanon
}

\section{Key Words}

Indocyanine green · Retinoblastoma - Thermotherapy

\begin{abstract}
Purpose: To report the outcome of pediatric patients with retinoblastoma refractory to traditional local therapy who were treated with indocyanine green (ICG)-enhanced thermotherapy. Materials and Methods: This is a retrospective review of a case series of 3 patients with bilateral retinoblastoma who were treated with ICG-enhanced thermotherapy after showing no response to conventional chemothermotherapy or transpupillary thermotherapy (TTT) alone noted on two consecutive examinations under anesthesia. Results: The 3 patients had had one eye enucleated previously due to advanced disease, and the remaining eye was diagnosed with a large tumor, which showed either a marginal or no response to systemic chemotherapy and TTT. Addition of ICG enhancement during the subsequent TTT session shrunk the tumor to a measurable size that could then be followed by TTT alone as a means of treatment. One patient had tumor recurrence, at which time additional TTT without ICG was successfully applied after the tumor size had decreased; ICG enhancement was then added whenever TTT alone provided no response. Conclusions: ICG enhancement with TTT led to a measurable tumor regression in lesions that had previously not been responsive to traditional chemothermotherapy or isolated TTT. Message: These tumors had shown a minimal to no response to previous TTT treatment. However, adding ICG resulted in a measurable regression even though the same TTT treatment parameters were applied.




\section{Introduction}

Retinoblastoma is the most common primary ocular tumor in children and has a high survival rate in industrialized countries ranging from 86 to $92 \%$ [1-2]. Several conservative treatment modalities have been evaluated with different success rates. Transpupillary thermotherapy (TTT) was shown to be effective in small- and moderate-sized tumors and permitted globe salvage in combination with chemotherapy in $84 \%$ of cases classified as Reese-Ellsworth groups I-IV and in $47 \%$ of cases classified as group V [3].

The optimal photothermal effect of laser treatment can be obtained when lasers of appropriate wavelength are used in conjunction with the matching chromophore [4]. Several studies reported enhanced laser-tumor tissue photothermal interaction between the diode laser used in TTT $(810 \mathrm{~nm})$ and indocyanine green (ICG) dye and showed that ICG could decrease the laser fluence and irradiance threshold in TTT photocoagulation properties [5, 6]. As a result, this treatment has been used in other ocular conditions such as ocular melanoma and choroidal neovascularization [7, 8]. A recent case series showed that ICG-enhanced TTT combined with ophthalmic artery chemosurgery could be effective in retinoblastoma refractory to conventional laser treatment [9].

In this series, we present 3 patients with retinoblastoma refractory to standard TTT and intravenous chemotherapy who underwent ICG-enhanced TTT.

\section{Materials and Methods}

This is a retrospective review of the medical records of 3 patients with retinoblastoma who presented to the Children Cancer Center of Lebanon at the American University of Beirut Medical Center between March 2012 and December 2013. All patients included in this series were diagnosed with bilateral retinoblastoma, and received chemothermotherapy, which consisted of vincristine, etoposide, and carboplatin (VEC) followed by TTT within $24 \mathrm{~h}$. A total of 6 cycles of VEC were given. In refractory cases, 2 additional cycles of VEC or second-line chemotherapy with vincrisitine and topotecan were given, as recommended for each case after a multidisciplinary discussion. Photographic documentation was obtained with the RetCam machine (Massie Industries, Dublin, Calif., USA) at every eye examination under anesthesia (EUA).

Demographic data collected included age, sex, treatment status, number of chemotherapy cycles and the timing and number of TTT sessions. Tumor data included international classification, largest tumor basal diameter, location, indication for TTT, treatment parameters, and regression pattern.

Treatment was performed during EUA and delivered via an indirect ophthalmoscope-equipped infrared diode laser at $810 \mathrm{~nm}$ (Iridex, Mountain View, Calif., USA), focused with a 20- or 28-diopter lens. Duration and power of treatment were increased until a gray-white discoloration appeared. ICG-enhanced TTT was done when the lesion showed no reduction in size after conventional TTT on RetCam images after 2 consecutive EUAs 3-4 weeks apart. ICG dye $(0.6 \mathrm{mg} / \mathrm{kg})$ was delivered intravenously $30 \mathrm{~s}$ before laser treatment. Follow-up examinations occurred every 3-4 weeks and the need for additional treatment was decided based on the clinical appearance of the tumors and the regression pattern.

\section{Results}

\section{Case 1}

A 9-month-old boy was diagnosed with bilateral retinoblastoma with group $\mathrm{E}$ disease in the right eye and group B tumor in the left eye, as per the international classification of retinoblastoma [9]. He underwent enucleation of the right eye. Initial fundus examination of the left eye revealed a large tumor in the posterior pole just inferior to the optic nerve measuring $7 \mathrm{~mm}$ in basal diameter, and 3 other smaller lesions in the inferior periphery (fig. 1a). 
Ocular Oncology

and Pathology

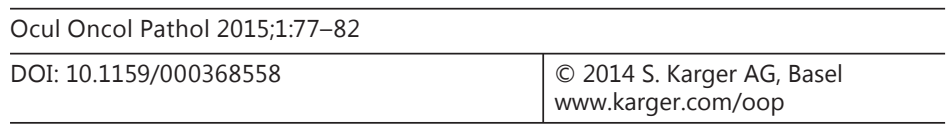

Al-Haddad et al.: Indocyanine Green-Enhanced Thermotherapy for Retinoblastoma
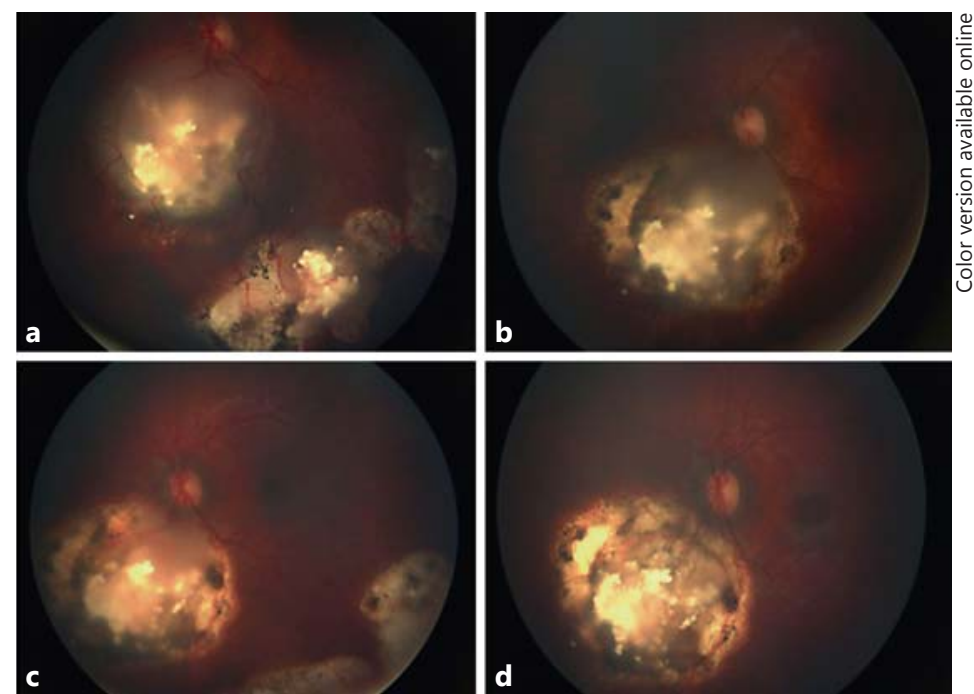

Fig. 1. Tumor regression pattern of case 1: at the second cycle of chemotherapy (a), after 6 cycles of chemothermotherapy (b), 6 weeks after receiving ICG-enhanced TTT (c), and after 4 weeks of the second ICG-enhanced TTT (d).

The patient received 6 cycles of intravenous chemotherapy (VEC) in combination with a total of 6 cycles of TTT to the large tumor in the posterior pole with a mean power of 700 $\mathrm{mW}(600-800)$ and a mean treatment duration of $5 \mathrm{~min}$ at every session. In addition, 4 cycles of cryotherapy were performed to the peripheral lesions. After 6 months of treatment, the peripheral lesions responded to treatment and calcified, but the large tumor in the posterior pole did not show improvement on more than 2 consecutive examinations (fig. 1b).

ICG-enhanced TTT was then performed with laser power ranging from 600 to $800 \mathrm{~mW}$ for a total of 6 min of treatment. After 4 weeks of follow-up, a measurable improvement was noticed (fig. 1c). Further, monthly TTT was performed due to residual active disease, and after 3 sessions of TTT (over 3 months) another session of ICG-enhanced TTT was performed. Four weeks later, the tumor shrunk significantly and became flatter with minimal activity (fig. 1d). On the last follow-up, 5 weeks later, the lesion remained quiescent, and no further treatment was applied.

\section{Case 2}

A 14-month-old girl was diagnosed with bilateral retinoblastoma. She had undergone enucleation of the left globe before presenting to our hospital. Fundus examination of the right eye revealed a large tumor (group B) measuring about 8-9 $\mathrm{mm}$ in basal diameter occupying the posterior pole in the macula with calcifications and 2 smaller peripheral lesions (fig. 2a). The patient received 8 cycles of VEC in combination with TTT with laser power ranging from 800 to $900 \mathrm{~mW}$, and a total duration of treatment ranging between 6 and $10 \mathrm{~min}$ in each session. The peripheral lesions responded to therapy and became scarred, but the large tumor in the posterior pole showed a slow response (fig. 2b). ICG-enhanced TTT was used at the time of the last chemotherapy session with laser power ranging from 700 to 900 $\mathrm{mW}$ and a total duration of $10 \mathrm{~min}$. The tumor regressed in size such that it was possible to administer 2 more sessions of TTT without ICG after 2 months. This led to further improvement and the tumor regressed completely (fig. 2c).

Case 3

A 3-month-old girl was diagnosed with bilateral retinoblastoma with group $\mathrm{E}$ in the right eye and group B in the left eye. She underwent enucleation of the right eye. Fundus 
Ocular Oncology

and Pathology
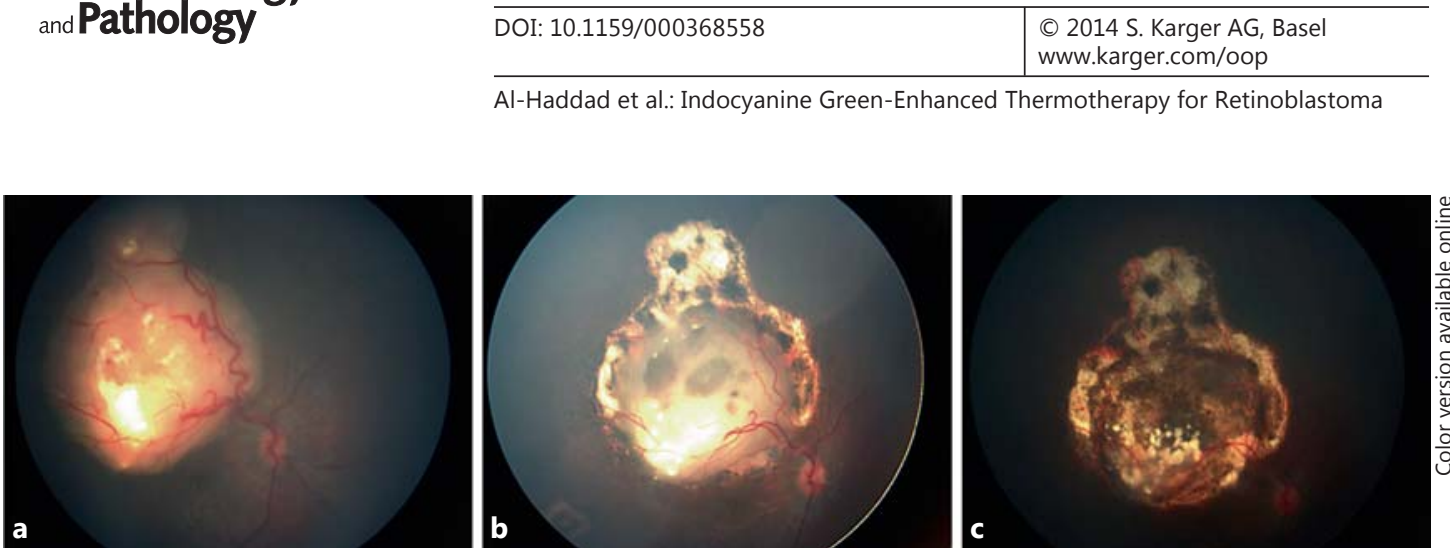

Fig. 2. Tumor regression pattern of case 2: initial tumor size before any treatment (a), after 6 cycles of chemothermotherapy (b), and 8 weeks after receiving ICG-enhanced TTT (c).
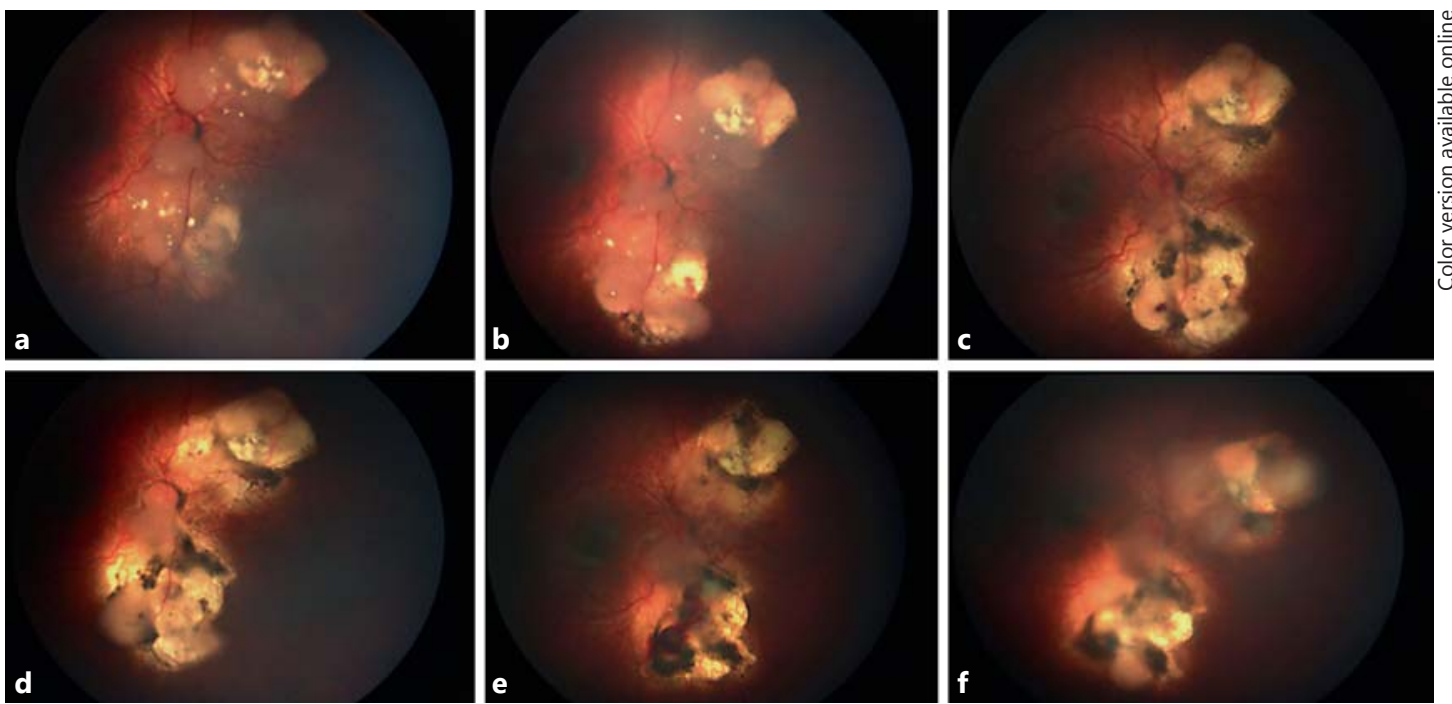

Fig. 3. Tumor regression pattern of case 3: at presentation (a), after 5 sessions of chemothermotherapy (b), 4 weeks after receiving ICG-enhanced TTT (c), partial tumor response after 2 sessions of TTT alone (d), 4 weeks after receiving additional ICG-enhanced TTT (e), and on the last examination (f).

examination of the left eye revealed 2 large tumors next to the optic nerve measuring 8-10 $\mathrm{mm}$ each in basal diameter. Six cycles of VEC were given in combination with TTT. Partial response was noted with residual lesions next to the optic nerve superiorly and inferiorly measuring 3 disc diameters each (fig. 3a). The patient received 2 additional cycles of chemotherapy (vincristine and topotecan) with TTT treatment without improvement (fig. 3b). During the last cycle of topotecan therapy, ICG-enhanced TTT was performed with a total power of 800-900 $\mathrm{mW}$ for a total of $10 \mathrm{~min}$. After 4 weeks of follow-up, an improvement was noted with a prominent decrease in size and thickness of the tumor (fig. 3c). The patient received 4 additional TTT sessions without using ICG every 3-4 weeks of follow-up, but a suboptimal response was again noted (fig. 3d). Hence, ICG-enhanced TTT was repeated and a measurable decrease in tumor size and thickness were noted on follow-up examination with residual activity at the optic nerve (fig. 3e). The lesion increased in size again on subsequent EUA, and 2 sessions of ICG-enhanced TTT were administered. On the last examination, the tumor had not resolved completely (fig. $3 \mathrm{f}$ ) and other treatment options are being undertaken. 


\section{Ocular Oncology \\ and Pathology}

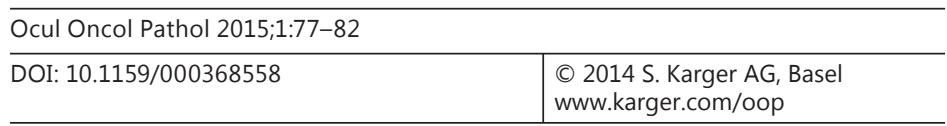

Al-Haddad et al.: Indocyanine Green-Enhanced Thermotherapy for Retinoblastoma

\section{Discussion}

A measurable regression of retinoblastoma refractory to conventional treatment was observed in our series when ICG dye was administered prior to thermotherapy. These tumors had shown minimal to no response to previous TTT treatment. However, adding ICG provided a measurable regression even though the same TTT treatment parameters were applied. Only 1 report in the literature examined the role of ICG enhancement to TTT in retinoblastoma. Francis et al. [10] reported that ICG-enhanced TTT in combination with ophthalmic artery chemosurgery effectively treated retinoblastoma in 13 patients who were unresponsive to conventional therapy without inducing retinal toxicity as documented by electroretinography. They postulated that 2 mechanisms were responsible for the added treatment effect: the thermal effect, which was directly cytotoxic, and the photodynamic effect, which generated reactive oxygen [10].

Two of our patients were treated with ICG-enhanced TTT at the time of their last intravenous chemotherapy cycle (cases 1 and 2), but these 2 patients had not shown response to prior chemothermotherapy. One patient received ICG-enhanced TTT while off chemotherapy (case 3). The patients served as their own controls; after ICG administration, the same TTT parameters were applied as those performed without ICG. A measurable regression of the tumor lasting more than 3 months was noted in the first 2 cases. Case 3 had disease recurrence despite early improvement, which could be attributed to the larger tumor size and the proximity to the optic nerve. Furthermore, underlying tumor biology differences may also have contributed to the differing responses among the described cases. The ICG-enhanced treatments helped reduce the tumor size until it was closer to the retinal pigment epithelium so that laser energy could be absorbed more efficiently. This made further TTT without ICG effective against these lesions.

Retinoblastoma lesions are usually nonpigmented and may be too large or too thick for TTT treatment alone. In addition, treatment modalities such as radiation can lead to chorioretinal scarring which makes the absorption of the laser energy even more difficult if attempting TTT alone. Enhancement with ICG may be particularly helpful in those situations. This dye has a high molecular weight and tends to be retained intravascularly [11]. The main aim behind the use of ICG in these situations would be to make the tumor tissue containing ICG more sensitive to the thermal therapy delivered by the infrared lasers. Moreover, ICG may allow the desired clinical endpoint with reduced laser parameters [6].

In conclusion, enhancement with ICG provides yet another option in treating large or well-differentiated retinoblastomas that are unresponsive to conventional thermotherapy allowing globe and vision salvage. This could be applied as an independent modality and not necessarily with intravenous or ophthalmic artery chemotherapy. ICG has been shown to be safe in children and has been used in a number of applications in the medical field including cardiovascular, hepatic, and ophthalmic conditions $[7,8,12]$. This work confirms previous findings that demonstrate the efficacy of ICG/TTT, but future studies are still needed to select the optimal dose of the dye, time between ICG and TTT, duration and power of laser needed and the long-term rate of recurrence/tumor control after adjuvant ICG.

\section{Disclosure Statement}

The authors declare that they have no conflicts of interest. 


\section{References}

1 Shields JA, Shields CL: Management and prognosis of retinoblastoma; in Shields JA, Shields CL (eds): Intraocular Tumors: An Atlas and Textbook. Philadelphia, WB Saunders Co, 1992, pp 377-391.

2 Gatta G, Capocaccia R, Coleman MP, Ries LA, Berrino F: Childhood cancer survival in Europe and the United States. Cancer 2002; 95:1767-1772.

-3 Shields CL, Honavar SG, Meadows AT, Shields JA, Demirci H, Singh A, Friedman DL, Naduvilath TJ: Chemoreduction plus focal therapy for retinoblastoma: factors predictive of need for treatment with external beam radiotherapy or enucleation. Am J Ophthalmol 2002;133:657-664.

4 Chen WR, Adams RL, Heaton S, Dickey DT, Bartels KE, Nordquist RE: Chromophore-enhanced laser-tumor tissue photothermal interaction using a 808-nm diode laser. Cancer Lett 1995;88:15-19.

-5 Chong LP, Ozler SA, de Queiroz JM Jr, Liggett PE: Indocyanine green-enhanced diode laser treatment of melanoma in a rabbit model. Retina 1993;13:251-259.

6 Peyman GA, Genaidy M, Yoneya S, Men G, Ghahramani F, Kuo PC, Bezerra Y, Nishiyama-Ito Y, Moshfeghi AA: Transpupillary thermotherapy threshold parameters: effect of indocyanine green pretreatment. Retina 2003; 23:378-386.

7 De Potter P, Jamart J: Adjuvant indocyanine green in transpupillary thermotherapy for choroidal melanoma. Ophthalmology 2003;110:406-413.

-8 Flower RW: Experimental studies of indocyanine green dye enhanced photocoagulation of choroidal neovascularization feeder vessels. Am J Ophthalmol 2000;129:501-512.

-9 Novetsky D, Abramson D, Kim J, Dunkel I: Published international classification of retinoblastoma (ICRB) definitions contain inconsistencies - an analysis of impact. Ophthalmic Genet 2009;30:40-44.

10 Francis JH, Abramson DH, Brodie SE, Marr BP: Indocyanine green enhanced transpupillary thermotherapy in combination with ophthalmic artery chemosurgery for retinoblastoma. Br J Ophthalmol 2013;97:164-168.

11 Owens SL: Indocyanine green angiography. Br J Ophthalmol 1996;80:263-266.

12 Kogon B, Fernandez J, Kanter K, Kirshbom P, Vincent B, Maher K, Guzetta N: The role of intraoperative indocyanine green fluorescence angiography in pediatric cardiac surgery. Ann Thorac Surg 2009;88:632-636. 